\title{
MED12 mutation in patients with hysteromyoma
}

\author{
JUN YE, HUA WANG, YA-BAO CHEN, DONG-LAN YUAN, LI-XIN ZHANG and LIN LIU \\ Department of Laboratory Medicine, Taizhou People's Hospital, Taizhou, Jiangsu 225300, P.R. China
}

Received May 13, 2014; Accepted January 22, 2015

DOI: $10.3892 / \mathrm{ol} .2015 .3118$

\begin{abstract}
Mediator Complex Subunit 12 (MED12) is a subunit of the mediator complex, which is believed to regulate global, as well as gene-specific, transcription. It has been reported that MED12 is mutated at high frequency in hysteromyoma. Recent studies have also shown that MED12 presents with different mutation frequencies in hysteromyoma patients of different populations. However, there are few studies with regard to the MED12 gene mutation in hysteromyoma patients in the Chinese Han population. In the present study, the MED12 mutations of 171 patients with hysteromyoma were analyzed; the results showed that 93 patients exhibited different MED12 mutations, including $131 \mathrm{G} \rightarrow \mathrm{T}, 131 \mathrm{G} \rightarrow \mathrm{A}, 130 \mathrm{G} \rightarrow \mathrm{A}, 146 \mathrm{C} \rightarrow \mathrm{T}, 130 \mathrm{G} \rightarrow \mathrm{A}, 130 \mathrm{G} \rightarrow \mathrm{C}$, $128 \mathrm{~A} \rightarrow \mathrm{C}, 130 \mathrm{G} \rightarrow \mathrm{T}, 127 \mathrm{Ins} 27,118$ _132Del15, 117_134Del18, 131_148Del18 and 141_165Del15. The mutation frequency was similar to that found in individuals of African descent or individuals of other non-Caucasian ethnicities, and lower than that in the Finnish or North American populations. Further analysis of 141 patients whose hysteromyoma was measured showed that the mutation frequency of MED12 in patients with large hysteromyomas was significantly lower than that in those with small hysteromyomas. These results suggested that MED12 mutation was important in the development of hysteromyomas in the Chinese Han population and that the size of the hysteromyoma may negatively correlate with the mutation frequency of MED12. This study supplemented current information on MED12 mutations in different races and may aid in developing personalized diagnoses for patients with hysteromyoma in the future.
\end{abstract}

\section{Introduction}

Hysteromyoma is a common disease and its incidence in females $>45$ years old is $>60 \%$ (1). Hysteromyomas are monoclonal tumors that arise from the uterine myometrium

Correspondence to: Dr Hua Wang, Department of Laboratory Medicine, Taizhou People's Hospital, 210 Yingchun Road, Taizhou, Jiangsu 225300, P.R. China

E-mail: yejun20110217@gmail.com

Key words: Chinese Han population, hysteromyoma, mediator complex subunit 12 , mutation, race smooth muscle cells. The tumors are estrogen- and progesterone-dependent and occur in reproductive females, usually prior to the menopause. It has been reported that the incidence of hysteromyoma is $5.1 \%$ in Chinese females who are 25-30 years old (2). Although hysteromyomas are benign, the tumors are able to trigger a number of diseases and cause severe complications, including abnormal uterine bleeding, abdominal pain and discomfort, pregnancy complications and even infertility (3). The common consequence of a hysteromyoma is a hysterectomy, which gives rise to social and economic problems $(4,5)$.

The protein coded by mediator complex subunit 12 (MED12) is a subunit of the mediator complex, which is a transcription factor that regulates gene expression and consists of 26 subunits (6). Certain recent studies have shown that MED12 presents with different mutations frequencies in hysteromyoma patients of different racial populations (7-9), which suggests that MED12 mutations have an important role in causing hysteromyoma. At present, there is no study with regard to MED12 gene mutations in hysteromyoma patients of the Chinese Han population.

In the present study, the biopsy specimens of 171 patients with hysteromyoma were analyzed for SNP mutations of MED12, and then the mutation frequency was compared with that of other races. The association between the size of the hysteromyomas and the MED12 mutation frequency was also analyzed.

\section{Materials and methods}

Eligibility and patient consent. All patients who met the inclusion criteria were provided with detailed information about the present study and their rights by the principal investigator/s. Han Chinese patients, aged between 30 and 65 years, with pathologically confirmed hysteromyoma were considered eligible for the study. The patients who were willing to take part provided written informed consent and were enrolled into the study. This study was approved by the ethics committee of The Taizhou People's Hospital (Taizhou, China) and all experiments were performed in accordance with the China Food and Drug Adminstration Guidelines for Ethical Review Work of Drug Clinical Trials (10).

Materials. In total, 171 hysteromyoma biopsy samples were obtained from in-hospital patients undergoing surgery in the Department of Obstetrics and Gynecology of The Taizhou People's Hospital. Among these patients, 13 cases had samples 
A

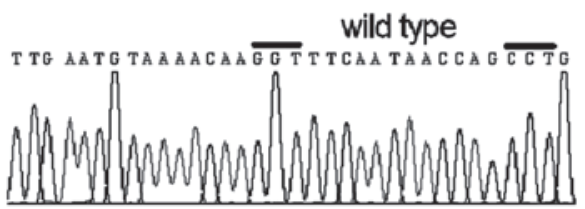

C

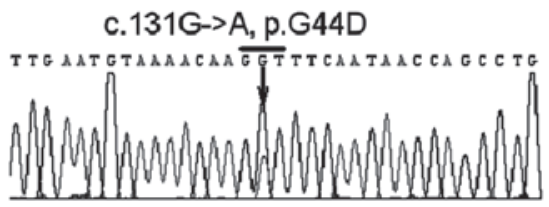

$\mathbf{E}$

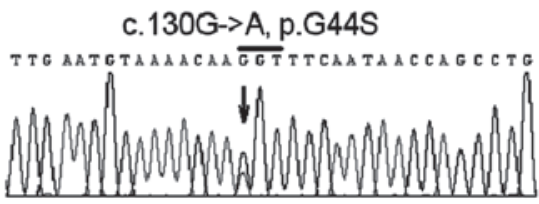

G

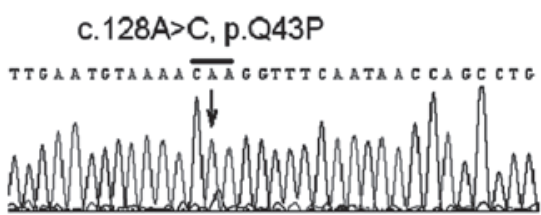

B

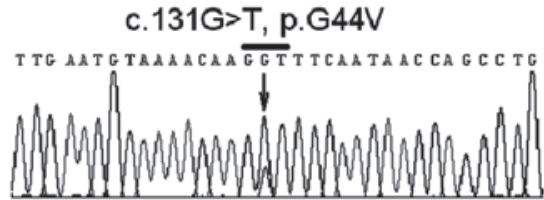

D

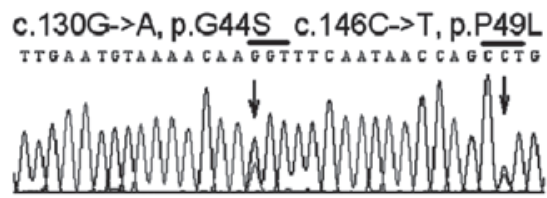

$\mathbf{F}$

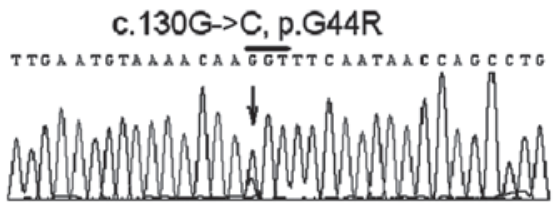

H

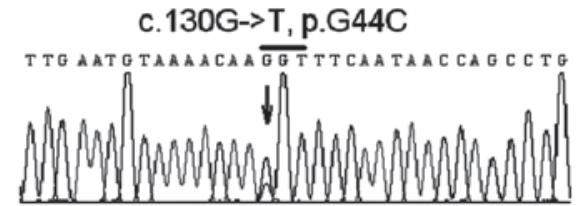

Figure 1. Sequence chromatograms showing somatic mutations in the second exon of MED12 in uterine hysteromyoma patients from the Chinese Han population. (A) Wild-type and (B-H) mutated sequences are shown. Mutated bases are indicated by arrows.

taken from the tissues surrounding the hysteromyoma. All samples were fresh tissues and were frozen at $-20^{\circ} \mathrm{C}$ for later analysis.

\section{Amplification of the second exon in MED12}

Design and synthesis of primers. According to the references and sequence found in the National Center for Biotechnology Information (NCBI) GenBank (NCBI sequence, NG_012808.1), the following primers were designed: MED12-F, 5'-GCCCTTTCACCTTGTTCCTTC-3' and MED12-R, 5'-GTCCCTATAAGTCTTCCCAAC-3'.

Genome isolation. Hysteromyoma biopsy tissues or biopsy tissues from the surrounding hysteromyoma were placed separately into mortars precooled by ice, and were then ground quickly into homogenates in liquid nitrogen using a pestle. Next, the genomes were isolated using the Tissue Genome Isolation kit, containing $50 \mathrm{mM}$ Tris-HCl lysis buffer (150 mM NaCl, 1 mM EDTA, 1\% Triton X100, 1\% sodium deoxycholate and $0.1 \%$ SDS; pH 7.4), $100 \mathrm{mg} / \mathrm{ml}$ RNaseI and $20 \mathrm{mg} / \mathrm{ml}$ protein $\mathrm{K}$ [Tiangen Biotech (Beijing) Co., Ltd., Beijing, China], according to the manufacturer's instructions. The homogenates were lysed in Tris- $\mathrm{HCl}$ lysis buffer and RNA was digested using $4 \mu \mathrm{l}$ RNaseI. The protein was then digested using $20 \mu \mathrm{l}$ protein $\mathrm{K}$. Next, $200 \mu \mathrm{l}$ ethanol was added to the sample and centrifuged at 13,400 x $\mathrm{g}$ for $60 \mathrm{sec}$, using the TIANamp Spin Column CB3 (Tiangen Biotech (Beijing) Co., Ltd.). TIANamp Spin Column CB3 was placed into the $1.5 \mathrm{ml}$ microcentrifuge tube, $700 \mu \mathrm{l}$ of wash buffer (75\% ethanol) was added, and centrifuged at 13,400 x g for 30 s at room temperature. subsequently an additional $500 \mu \mathrm{l}$ PW wash buffer (75\% ethanol) was added and centrifuged at $13,400 \times \mathrm{g}$ for a further $3 \mathrm{~min}$ at room temperature. Finally, the TIANamp Spin Column CB3 was placed into a new clean collection tube and $100 \mu \mathrm{l}$ distilled water was added to elute the genomic DNA, was incubated at room temperature $\left(15-25^{\circ} \mathrm{C}\right)$ for $5 \mathrm{~min}$ and subsequently centrifuged at $13,400 \mathrm{x} \mathrm{g}$ for $2 \mathrm{~min}$ at room temperature. The eluent, which included the genomic DNA, was then collected.

PCR amplification and sequencing of the MED12 gene. The PCR reaction system consisted of the following: $5 \mu 1$ genome template (30 ng), $5 \mu 110 \mathrm{X}$ tag buffer, $2.5 \mathrm{mM}$ dNTP mix (Futaibio Technology Company, Taizhou, China) and Taq polymerase (Futaibio Technology Company). PCR was performed using the GeneAmp PCR system 9700 (Applied Biosystems Life Technologies, Foster City, CA, USA). The PCR conditions were as follows: Pre-denaturation at $95^{\circ} \mathrm{C}$ for $5 \mathrm{~min}$, followed by 35 cycles of $94^{\circ} \mathrm{C}$ for $30 \mathrm{sec}, 55^{\circ} \mathrm{C}$ for $30 \mathrm{sec} 72^{\circ} \mathrm{C} 30 \mathrm{sec}$ and then $72^{\circ} \mathrm{C}$ for $5 \mathrm{~min}$. The obtained PCR amplification products were sequenced by Nanjing Jinsirui Biotechnology Co., Ltd. (Nanjing, China).

Statistical analysis. SigmaPlot version 12.0 (Systat Software, Inc., San Jose, CA, USA) was used for the statistical analysis, and the comparison of mutation rate was analyzed by $\chi^{2}$ test. 
$\mathrm{P}<0.05$ was considered to indicate a statistically significant difference.

\section{Results}

Sequence of the second exon in the MED12 gene of the hysteromyoma and adjacent tissue samples. The sequencing results from the 171 hysteromyoma tissue samples revealed 93 cases $(54.39 \%)$ with mutations. The tissues surrounding the hysteromyoma did not present with mutations (Fig. 1A). Among the mutations in the hysteromyoma tissues, $23(13.45 \%)$ presented as $131 \mathrm{G} \rightarrow \mathrm{T}$ (Fig. 1B), $17(9.94 \%)$ presented as $131 \mathrm{G} \rightarrow \mathrm{A}$ (Fig. 1C), 5 (2.92\%) presented as $130 \mathrm{G} \rightarrow \mathrm{A}$ and $146 \mathrm{C} \rightarrow \mathrm{T}$ (Fig. 1D), 6 (3.51\%) presented as $130 \mathrm{G} \rightarrow \mathrm{A}$ (Fig. 1E), $7(4.09 \%)$ presented as $130 \mathrm{G} \rightarrow \mathrm{C}$ (Fig. 1F), $6(3.51 \%)$ presented as $128 \mathrm{~A} \rightarrow \mathrm{C}($ Fig. $1 \mathrm{G})$ and $11(6.43 \%)$ presented as $130 \mathrm{G} \rightarrow \mathrm{T}$ (Fig. $1 \mathrm{H})$. In addition to these SNP mutations, there were 3 insertion mutations (1.75\%; 127Ins27) and 15 deletion mutations $(8.77 \%$; two cases of 118_132Del15, three cases of 117_134Del18, three cases of 131_148Del18 and seven cases of 141_165Del15). Among these mutations, 146C $\rightarrow$ T, 127Ins27, 135Del46, 118Del15, 141Del15 and 117Del18 have been reported for the first time by the present study.

Comparison of the second exon in the MED12 gene in the Chinese Han population versus populations of other ethnicities. Certain studies have reported that the mutation frequency of the second exon of MED12 in patients with hysteromyoma was $70.67 \%(159 / 225)$ in the Finnish population (8), 50.0\% (14/28) in individuals of African descent or individuals of other non-Caucasian ethnicities (7), and 67\% (100/147) in North American populations (9). The present study showed that the mutation frequency of the second exon of the MED12 gene in the hysteromyoma patients in the present Chinese Han population was $54.39 \%$, which was significantly different from mutation frequencies in the Finnish or North American $\left(\chi^{2}=10.437, \mathrm{P}=0.001 ; \chi^{2}=5.607, \mathrm{P}=0.018\right)$ populations, but not significantly different from that of the individuals of African descent or individuals of other non-Caucasian ethnicities $\left(\chi^{2}=0.0516, \mathrm{P}=0.820\right)$.

To detect the association between the size of the hysteromyoma tissue sample and the mutation frequency, samples from 141 hysteromyoma patients whose tumor sizes were measured underwent analysis. The results showed that 33 out of the 84 cases with a hysteromyoma of $\geq 5 \mathrm{~cm}$ in diameter presented with MED12 mutations (39.29\%), and 34 out of the 57 cases with a hysteromyoma of $<5 \mathrm{~cm}$ in diameter presented with MED12 mutations (59.65\%). These two rates were significantly different $\left(\chi^{2}=4.859, \mathrm{P}=0.0027\right)$.

\section{Discussion}

Difference in ethnicity is an important risk factor in epidemiology, particularly for hysteromyoma. Certain studies have shown that the incidence of hysteromyoma varies among populations of different ethnicities (11-16). For example, the incidence of hysteromyoma in African females is three times as high as that in Caucasian females (13). Also, compared with other ethnicities, African females with hysteromyoma are younger and have larger lesions, with hysteromyomas that grow faster $(12,15)$. The incidence of hysteromyoma in females of African descent is higher than in individuals of other ethnicities, including Hispanic and Asian individuals $(11,15,16)$. Certain studies have indicated that other risks, such as family history, smoking, drinking, hypertension and weight increase, can increase the risk of hysteromyoma, in addition to the risk associated with ethnicity $(12,14,16,17)$.

A study by Mäkinen et al (8) indicated that $70 \%$ of patients with hysteromyoma in the Finnish population presented with a mutation in the second exon of MED12, which affected the genetically-conserved sequence of the MED12 protein. Also, small myomas were shown to exhibit more mutations than large myomas. Another study by Mäkinen et al (7) detected mutations of the second exon in the MED12 genes from 28 pathological tissue samples obtained from 18 patients with hysteromyoma and reported a $50 \%$ mutation frequency rate. A study by McGuire et al (9) reported that the MED12 mutation frequency in North American females with hysteromyoma was $67 \%$ (100/148). In the present study, mutations of the second exon in the MED12 gene were analyzed in 171 pathological tissue samples in hysteromyoma patients, and the result showed that the mutation frequency was $54.39 \%$, which was significantly different from the Finnish and North American populations, but not significantly different from the population of individuals of African descent. Additionally, none of the adjacent tissue samples presented with MED12 mutations. The mutation frequency of $54.39 \%$ suggested that mutation in the second exon of the MED12 gene is also a significant risk factor for hysteromyoma in the Chinese Han population. Furthermore, the present study was the first to find several novel mutation types in the second exon of the MED12 gene of hysteromyoma patients.

Previous studies have shown that the second exon of the MED12 gene in hysteromyoma patients who were from Finland, North America or South Africa presented with high mutation frequencies (7-9). The reported mutation frequency in the South African population appeared to be lower than that found in Caucasian individuals, which may be a result of the size of the hysteromyoma in these patients. The tumors of the individuals of the South African population were usually large, and according the study by Mäkinen et al, the size of the hysteromyoma may be negatively correlated with the presence of mutations (7). In the present study, the mutation frequency of MED12 in hysteromyoma was $54.39 \%$, similar to that of the individuals of African descent, and lower than that of the Finnish or North American populations. Also, in the 141 hysteromyomas measured, the percentage of tumors with a diameter of $\geq 5 \mathrm{~cm}$ was $59.57 \%$ (84 cases), which was significantly higher than the percentage of tumors with a diameter of $<5 \mathrm{~cm}(40.43 \%$; 57 cases). This result suggested that patients in the Chinese Han population had larger hysteromyomas, which may be one of the reasons why the mutation frequency of the MED12 gene in the hysteromyoma patients of the Chinese Han population was lower than that of the Finnish or North American populations. The original Finnish study also reported that tumors lacking MED12 mutations tended to be larger $(\mathrm{P}=0.015)(8)$. However, it is not certain whether the myoma size in the Chinese population is actually larger on average compared with that of the Caucasian population, as the present study was only conducted on a small number of samples. Further studies must be performed to clarify this 
matter. In addition, the results of the present study also showed that the MED12 mutation frequency in small hysteromyomas was higher than that in large hysteromyomas, which was consistent with the study by Mäkinen et al (7).

The pathways for focal adhesion, extracellular matrix receptor interaction and Wnt signaling are altered in hysteromyomas with mutations (8). The association between the exact mutation of exon 2 in the MED12 gene and its consequent function has not been reported. In the present study, 13 mutations, namely $131 \mathrm{G} \rightarrow \mathrm{T}, 131 \mathrm{G} \rightarrow \mathrm{A}, 130 \mathrm{G} \rightarrow \mathrm{A}, 146 \mathrm{C} \rightarrow \mathrm{T}, 130 \mathrm{G} \rightarrow \mathrm{A}$, $130 \mathrm{G} \rightarrow \mathrm{C}, 128 \mathrm{~A} \rightarrow \mathrm{C}, 130 \mathrm{G} \rightarrow \mathrm{T}, 127 \mathrm{Ins} 27,118 \_132 \mathrm{Del15}$, 117_134Del18, 131_148Del18 and 141_165Del15, were found. All of these mutations were located in exon 2 of the MED12 gene. The association between each mutation and their consequent functions requires further study.

Although the present study was a pilot study, several novel mutation types were identified in the second exon of the MED12 gene in the studied hysteromyoma patients, and the study also suggested that mutations in the second exon of the MED12 gene also play a significant role in the development of hysteromyomas in the female Chinese Han population.

\section{Acknowledgements}

The authors would like to thank the staff of The Taizhou Peoples's Hospital for providing their support.

\section{References}

1. Okolo S: Incidence, aetiology and epidemiology of uterine fibroids. Best Pract Res Clin Obstet Gynaecol 22: 571-588, 2008.

2. Shi YF: Incidence, aetiology of uterine fibroids. Zhongguo Yi Kan 37: 11-12, 2002 (In Chinese)

3. Stewart EA: Uterine fibroids. Lancet 357: 293-298, 2001.

4. Flynn M, Jamison M, Datta S and Myers E: Health care resource use for uterine fibroid tumors in the United States. Am J Obstet Gynecol 195: 955-964, 2006.
5. Hartmann KE, Birnbaum H, Ben-Hamadi R, Wu EQ, Farrell MH, Spalding J and Stang P: Annual costs associated with diagnosis of uterine leiomyomata. Obstet Gynecol 108: 930-937, 2006.

6. Taatjes DJ: The human Mediator complex: a versatile, genome-wide regulator of transcription. Trends Biochem Sci 35: 315-322, 2010.

7. Mäkinen N, Heinonen HR, Moore S, Tomlinson IP, van der Spuy ZM and Aaltonen LA: MED12 exon 2 mutations are common in uterine leiomyomas from South African patients. Oncotarget 2: 966-969, 2011.

8. Mäkinen N, Mehine M, Tolvanen J, et al: MED12, the mediator complex subunit 12 gene, is mutated at high frequency in uterine leiomyomas. Science 334: 252-255, 2011.

9. McGuire MM, Yatsenko A, Hoffner L, Jones M, Surti U and Rajkovic A: Whole exome sequencing in a random sample of North American women with leiomyomas identifies MED12 mutations in majority of uterine leiomyomas. PLoS One 7: e33251, 2012.

10. Guidelines for Ethical Review Work of Drug Clinical Trials 2010. China Food and Drug Administration, Beijing, pp1-15, 2010.

11. Kjerulff KH, Langenberg P, Seidman JD, Stolley PD and Guzinski GM: Uterine leiomyomas. Racial differences in severity, symptoms and age at diagnosis. J Reprod Med 41: 483-490, 1996.

12. Marshall LM, Spiegelman D, Barbieri RL, et al: Variation in the incidence of uterine leiomyoma among premenopausal women by age and race. Obstet Gynecol 90: 967-973, 1997.

13. Baird DD, Dunson DB, Hill MC, Cousins D and Schectman JM: High cumulative incidence of uterine leiomyoma in black and white women: ultrasound evidence. Am J Obstet Gynecol 188: 100-107, 2003.

14. Wise LA, Palmer JR, Harlow BL, et al: Risk of uterine leiomyomata in relation to tobacco, alcohol and caffeine consumption in the Black Women's Health Study. Hum Reprod 19: 1746-1754, 2004.

15. Huyck KL, Panhuysen CI, Cuenco KT, et al: The impact of race as a risk factor for symptom severity and age at diagnosis of uterine leiomyomata among affected sisters. Am J Obstet Gynecol 198: 168.e1-168.e9, 2008.

16. Templeman C, Marshall SF, Clarke CA, et al: Risk factors for surgically removed fibroids in a large cohort of teachers. Fertil Steril 92: 1436-1446, 2009.

17. Wise LA, Palmer JR, Spiegelman D, Harlow BL, Stewart EA, Adams-Campbell LL and Rosenberq L: Influence of body size and body fat distribution on risk of uterine leiomyomata in U.S. black women. Epidemiology 16: 346-354, 2005. 